\title{
NISTIR 6322
}

\section{Gray Calibration of Digital Cameras to Meet NIST Mugshot Best Practice}

Mike Rubinfeld

Computer Scientist

Charles Wilson

Group Manager

U.S. DEPARTMENT OF COMMERCE Technology Administration

National Institute of Standards and Technology

Gaithersburg, MD 20899

QC
100
.$U 56$
$N 0.6322$
1999





\section{NISTIR 6322}

\section{Gray Calibration of Digital Cameras to Meet NIST Mugshot Best Practice}

\section{Mike Rubinfeld}

Computer Scientist

\section{Charles Wilson}

Group Manager

U.S. DEPARTMENT OF COMMERCE Technology Administration

National Institute of Standards and Technology

Gaithersburg, MD 20899

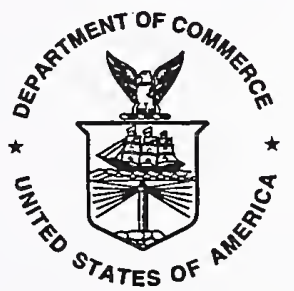

U.S. DEPARTMENT OF COMMERCE William M. Daley, Secretary

TECHNOLOGY ADMINISTRATION

Gary R. Bachula, Acting Under Secretary for Technology

NATIONAL INSTITUTE OF STANDARDS

AND TECHNOLOGY

Raymond G. Kammer, Director 



\section{Table of Contents}

ABSTRACT. 1

INTRODUCTION TO COLOR ELECTRONIC IMAGING 1

MUGSHOT PROBLEM .3

CONSTRAINTS. 4

EQUIPMENT USED FOR THE EXPERIMENT ................................................................................... 4

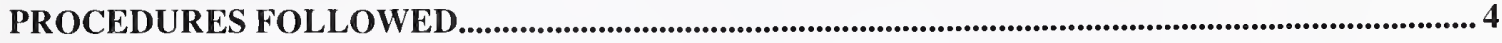

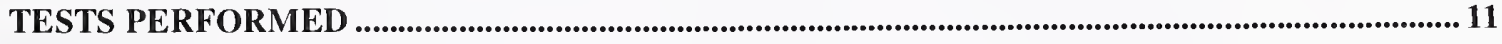

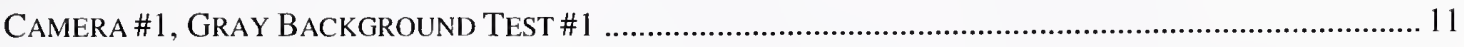

SONY CAMERA \#1, GRAY BACKGROUND TEST \#2 …................................................................................... 12

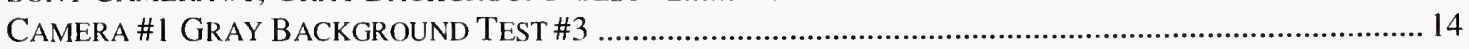

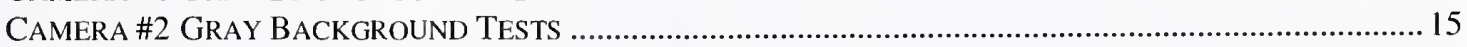

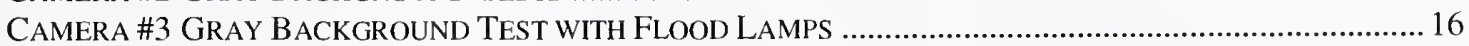

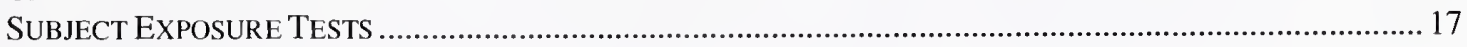

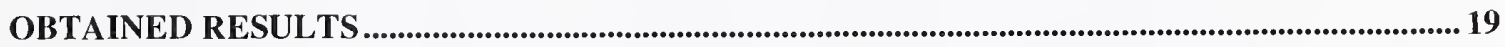

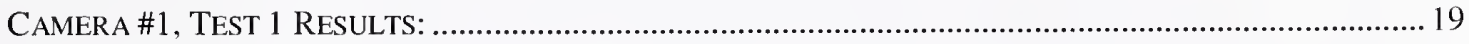

CAMERA \#1, TEST 2 RESULTS: ..................................................................................................... 20

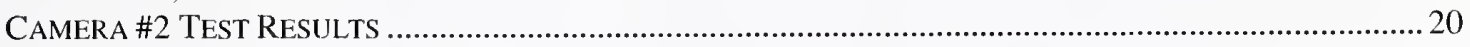

CONCLUSIONS AND INFERENCES ............................................................................................20

\section{Table References}

Table 1 - Macbeth Densitometer Readings........................................................................................................ 5

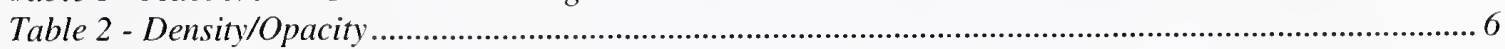

Table 3 - Results for Camera \#1, Test \#1 ............................................................................................... II

Table 4 - Results for Camera \#1, Test \#2 ……................................................................................... 13

Table 5 - Results for Camera \#1, Test \#3 ............................................................................................. 15

Table 6 - Results for Camera \#2 Test ................................................................................................. 15

Table 7 - Results for Camera \#3 Test ............................................................................................... 17

Table 8 - Camera \#1 Clothing Test Results with EV = 0.0 .................................................................. 18

Table 9 - Camera \#2 Clothing Test Results ................................................................................................ 19

Table 10 - Camera \#3 Clothing Test Results ............................................................................................. 19

\section{Figure References}

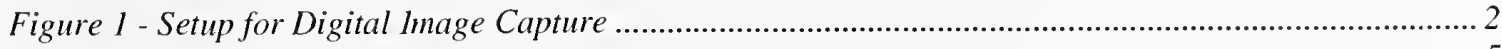

Figure 2- Sample 18\% Gray Patch........................................................................................................ 5

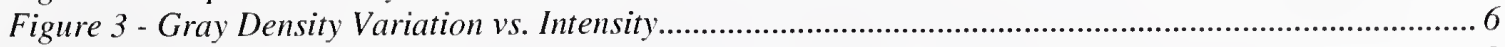

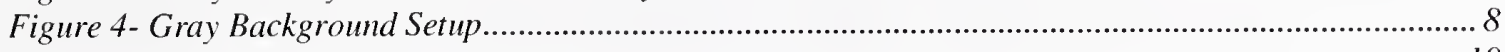

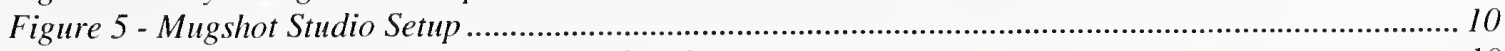

Figure 6 - Gray Level Values with Overhead Lights ............................................................................ 10

Figure 7 - Gray Level Values without Overhead Lights .......................................................................... 10

Figure 8 - Single Gray Sheet ................................................................................................................ 14 



\title{
Gray Calibration of Digital Cameras to meet NIST Mugshot Best practice
}

June 1, 1999

By Mike Rubinfeld, Computer Scientist

and

Charles Wilson, VIP Group Manager NIST

\begin{abstract}
A primary objective of any process of photography, electronic publishing, or printing is to reproduce all colors as perfectly as possible. A good starting point is to render a series of known shades of gray as accurately as possible. The goal of this experiment is to show how digital camera images can be calibrated to meet the NIST "Best Practice Recommendation for the Capture of Mugshots"1 (BPR) Document. This paper represents the results of a two-part experiment that was conducted to determine the best methodology for calibrating digital cameras for rendering gray images as close to the eighteen percent gray intensity level, specified for background color in the BPR document. The first part of the experiment was conducted to determine the characteristics of the specific cameras used in the experiment by measuring the rendered version of various known gray intensity levels and determining the amount of nonlinearity among the quantified gray samples. The second part of the experiment was a measurement of the effect that different colored clothing had on the rendered gray background for each of the three digital cameras used in the experiment. Results for three specific digital cameras are presented to illustrate the methods involved. These results will soon be out of date, because the technology is changing very rapidly.
\end{abstract}

\section{Introduction to Color Electronic Imaging}

The basic goal of photography, either film-based or digital, is to record a permanent image by capturing light reflected from a subject. In this experiment, several digital still cameras were used to capture images of gray test samples and mugshots against a gray background. These images were taken in an otherwise unlighted laboratory using lights with different intensity and color. The interpretation of the experimental results requires that the details of the digital image capture process be described by a physical model which is more complex than the simple three color models, RGB or CYMK, that are used to record and display the digital image in the computer.

\footnotetext{
${ }^{1}$ The NIST "Best Practice Recommendation for the Capture of Mugshots" document can be found at the NIST Web site with the link: http://www.nist.gov/itl/div894/894.03/face/bpr_mug3.html
} 
The experiment was further complicated by the fact that the CCD sensors used in these cameras were in part developed for applications where the goal was to produce imaging technology that was more sensitive than human vision; the cameras used here demonstrated this effect. The model is similar to the one that would be needed to characterize conventional color photography. The principal difference is in the capture process inside the camera. Techniques have been developed to insure consistent color quality for film-based image capture but these techniques are more complex than those presently in use for digital mugshot capture.

The physical processes that interact to generate the digital image are shown in Figure 1. Light from the three lamps, shown in the upper right of the figure, shines on
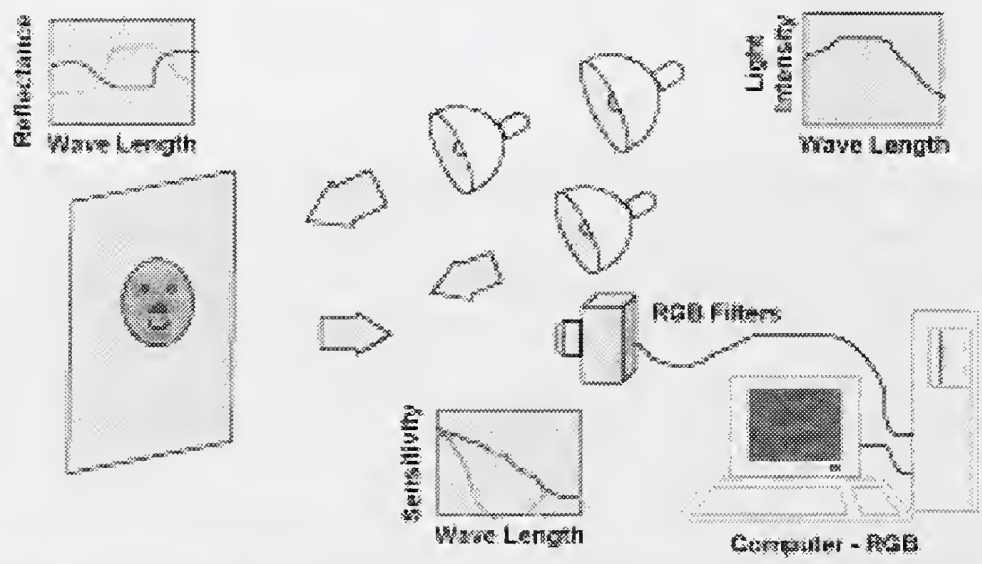

Figure 1 - Setup for Digital Image Capture

the subject shown on the left side of the figure and is captured by the digital camera connected to a computer shown in the lower right of the figure. Each of the processes relative to digital image capture is characterized by a wavelength dependent response, which affects the intensity of the light that is used by the camera to generate the final three-color image.

The lights have a wavelength dependent intensity illustrated by the graph in the upper right near the lamps. The shape of this curve affects all other aspects of the experiment and is controlled primarily by the color temperature of the lamps. The way a user can effect this color distribution is by the choice of light source. As the lamps age the intensity of illumination may also decrease.

The light from the lamps shines on the subject where it is reflected by two components, the gray background and the face of interest, which is illustrated by the blue mask. When the mask is removed from the camera field of view, the amount of light reflected by the background is a function of wavelength shown the black curve on the reflectance verses wavelength curve on the left-hand side of the figure. When the blue mask is in the field of view, the reflectance of the mask is illustrated by the blue curve in the graph. The mask does not affect the reflectance of the background. This should also be true for the digital image acquired by the computer, but, as we will discuss later, many 
low-end digital cameras contain automatic exposure controls, which adjust all exposures in a way that is dependent on the specific subject being imaged.

The camera captures the light from the subject. In the capture process, the three images are formed on a CCD array through red, green, and blue (RGB) color filters. One or more CCDs are used for the filtered images and the CCD has a wavelength dependent sensitivity which is illustrated by the black curve shown in the graph in the bottom center of the figure. This sensitivity is further changed by the three color filters illustrated by the red, green, and blue curves on the graph. The experiment presented here was carried out with digital cameras that have different color sensitivities. The measured sensitivity to light of different colors is the combined effect of the CCD sensor and the color filters used to generate the three-color image. Different digital cameras are manufactured using different CCD chips that have different wavelength sensitivity. These cameras also have color filters for three-color image separation that vary from one manufacture to another. This generates different color rendering in the final digital image which is similar to using different film types in film based cameras.

A user has a reasonably good chance of controlling the light intensity and of controlling the color temperature, thus affecting the uniform gray background reflectance. The internal parameters of different digital cameras are not under user control and are not specified by the camera manufacturers. The goal of this experiment is to use the factors that are under user control to measure the effect of unknown camera characteristics on the quality and uniformity of digital mugshot images.

\section{Mugshot Problem}

Both the BPR and the Mugshot Uniformity Guidelines states that a background of $18 \%$ gray intensity is required for mugshots. The physical background can be produced with little difficulty, especially if a standard $18 \%$ gray card (obtained from Eastman Kodak ${ }^{2}$ or other company involved in color standards) is available for a basis of comparison. The problem arises when a camera is used to render an image containing this background. The resultant image may or may not appear to have the same gray intensity level as the actual physical background. The resulting intensity value of the gray background will fluctuate due to various lighting conditions, exposure settings, CCD characteristics, clothing worn by the subject, etc. The problem becomes one of finding the best methodology to determine the appropriate camera settings to yield a rendering of the background that comes as close to the $18 \%$ gray as possible.

\footnotetext{
${ }^{2}$ Certain commercial imaging equipment or imaging software may be identified in order to adequately specify or describe the subject matter of this work. In no case does such identification imply recommendation or endorsement by the National Institute of Standards and Technology, nor does it imply that the equipment or software identified is necessarily the best available for the purpose.
} 


\section{Constraints}

The experiment was constrained to the use of digital cameras and the resultant digital images. We used a windowless office supplied with a constructed background that was painted to produce an eighteen-percent gray reflectance. We generated a set of twelve rectangular gray patches printed on letter-sized paper using the Adobe PhotoShop 4.0 software package. These gray patches were set at gray levels of 100 to 155 (on a scale of 0 to 255 where 0 represents total black and 255 represents total white) varying by five units of gray level for each gray patch. The reflectance of each gray patch was measured using a Macbeth color densitometer. This measurement allows the reflectance of the different gray samples to be compared using a method independent of the method used to generate the samples.

\section{Equipment Used for the Experiment}

1. Camera \#1 - 640x480 pixel resolution with fully automatic exposure control. The JPEG compression ratio was calculated to be approximately 24 to 1 . The EV settings ranged from +1.5 to -1.5 in incremental steps of 0.5 . The lens had a zoom ratio of $10 \mathrm{x}$.

2. Camera \#2 - 1024x768 pixel resolution with fully automatic exposure control. The JPEG compression ratio was calculated to be approximately 8 to 1 . The EV settings ranged from +2.0 to -2.0 in incremental steps of 0.5 . The lens had a zoom ratio of $3 \mathrm{x}$.

3. Camera \#3 - 1200x950 pixel resolution with both automatic exposure control and manual shutter and aperture override control. Compression was not used by this camera, and the lens could be zoomed from $50 \mathrm{~mm}$ to $80 \mathrm{~mm}$ focal length.

4. Tripod

5. Three floodlights

6. Photographic light meter

7. $18 \%$ reflectance gray backdrop wall

8. Macbeth color densitometer

9. PC with Adobe PhotoShop 4.0 installed

10. Epson 800 Color Stylus Inkjet Printer

\section{Procedures Followed}

A series of steps were followed for setting up the gray calibration experiment. These steps were as follows:

STEP 1: We prepared a set of 12 gray patches, one patch per image with the dimensions $19 \mathrm{~cm}$ by $25.4 \mathrm{~cm}$ for each image. Using Adobe's PhotoShop Version 4, the gray patches were set to varying gray levels from 100 to 155 with a delta of 5 gray level units between each gray patch. Each image was checked for accuracy by using the PhotoShop luminosity histogram function. Figure 2 is an example of a gray patch image with RGB values of 125,125 , and 125 respectively. 
STEP 2: Each gray patch image was printed out on the Epson 800 Color Stylus ink jet printer using Epson's ${ }^{2}$ special photographic quality A4 sized paper with the highest resolution setting of $1440 \mathrm{dpi}$. A first set of images was printed using the color setting, and a second set was printed using the black only setting.

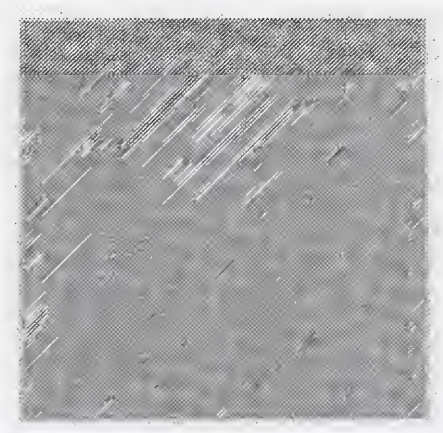

Figure 2- Sample 18\% Gray Patch

STEP 3: The output pictures from the Epson printer were then measured for gray density using a Macbeth Densitometer. Five measurements were taken at each of the four corners and the middle of each printed gray patch. The average value was then calculated for each gray patch. Table 1 shows all the densitometer average readings taken from the gray patch pictures along with the readings taken from the standard $18 \%$ gray card supplied by Kodak. Readings were taken for both the color setting and the "black-only" setting on the Epson printer. Through the use of the densitometer, we determined that the "black-only" setting gave results that most closely matched the Kodak gray Card density value. Figure 3 depicts the linear variation for the "black only" density readings as shown in Table 1. This result agreed with a visual comparison, more specifically, the 125 gray level value had a density value of 0.76 compared to the Gray Card value of 0.75 .

Table 1 - Macbeth Densitometer Readings

\begin{tabular}{|c|c|c|}
\hline Gray Level Value & Color Print Density & Black Only Print Density \\
\hline 100 & 0.63 & 1.00 \\
\hline 105 & 0.60 & 0.95 \\
\hline 110 & 0.59 & 0.89 \\
\hline 115 & 0.56 & 0.85 \\
\hline 120 & 0.54 & 0.80 \\
\hline 125 & 0.52 & 0.76 \\
\hline 130 & 0.51 & 0.74 \\
\hline 135 & 0.48 & 0.69 \\
\hline 140 & 0.45 & 0.67 \\
\hline 145 & 0.43 & 0.64 \\
\hline 150 & 0.40 & 0.61 \\
\hline 155 & 0.37 & 0.59 \\
\hline Kodak Gray Card & & 0.75 \\
\hline
\end{tabular}




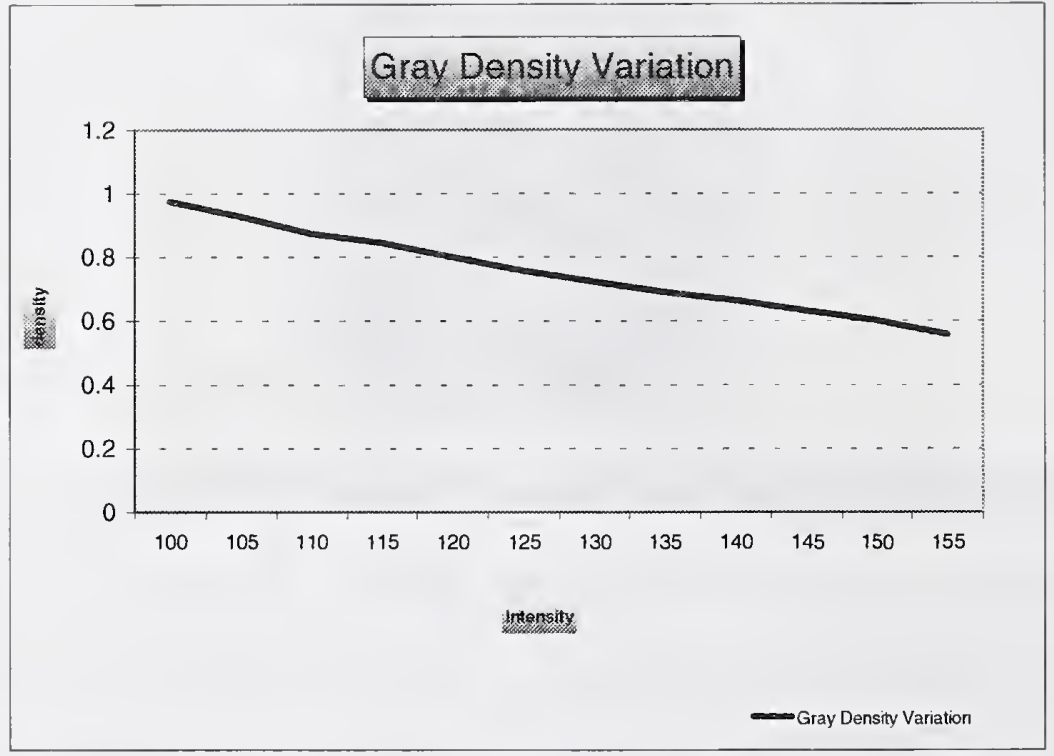

Figure 3 - Gray Density Variation vs. Intensity

STEP 4: The standard Density - Opacity Table as shown in Table 2 below was used as a basis of comparison for the values obtained from the Macbeth densitometer.

Table 2 - Density/Opacity

(Relating Transmission or Reflectance to Density)

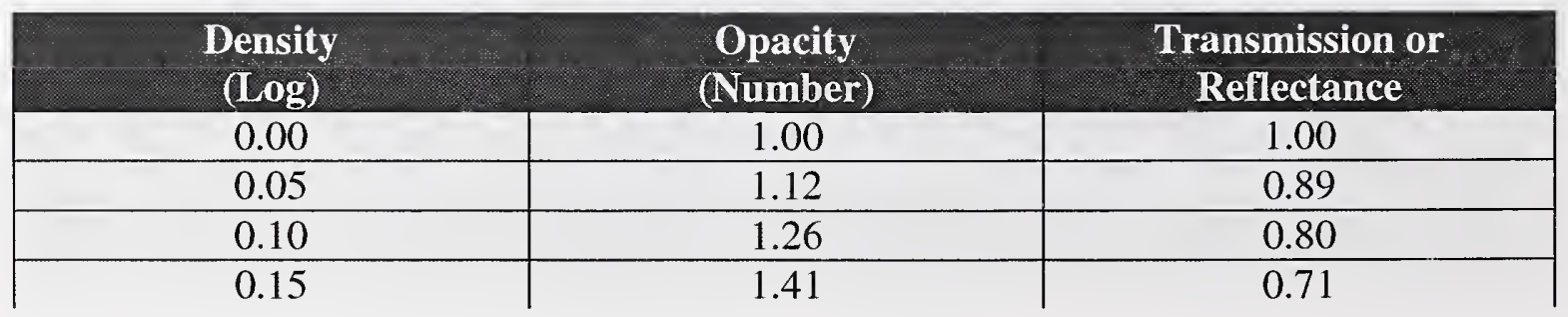




\begin{tabular}{|c|c|c|}
\hline $\begin{array}{c}\text { Density } \\
\text { (Log) }\end{array}$ & $\begin{array}{l}\text { Opacity } \\
\text { (Number) }\end{array}$ & $\begin{array}{c}\text { Transmission or } \\
\text { Reflectance }\end{array}$ \\
\hline 0.20 & 1.59 & 0.63 \\
\hline 0.25 & 1.78 & 0.56 \\
\hline 0.35 & 2.24 & 0.45 \\
\hline 0.45 & 2.82 & 0.36 \\
\hline 0.50 & 3.16 & 0.32 \\
\hline 0.55 & 3.55 & 0.28 \\
\hline 0.60 & 3.98 & 0.25 \\
\hline 0.65 & 4.47 & 0.22 \\
\hline 0.70 & 5.01 & 0.20 \\
\hline 0.75 & 5.62 & 0.18 \\
\hline 0.80 & 6.31 & 0.16 \\
\hline 0.85 & 7.08 & 0.14 \\
\hline 0.90 & 7.94 & 0.126 \\
\hline 0.95 & 8.91 & 0.112 \\
\hline 1.00 & 10.0 & 0.100 \\
\hline 1.05 & 11.2 & 0.089 \\
\hline 1.10 & 12.6 & 0.080 \\
\hline 1.15 & 14.1 & 0.071 \\
\hline 1.20 & 15.9 & 0.063 \\
\hline 1.25 & 17.8 & 0.056 \\
\hline 1.30 & 20.0 & 0.050 \\
\hline 1.35 & 22.4 & 0.045 \\
\hline 1.40 & 25.1 & 0.040 \\
\hline 1.45 & 28.2 & 0.036 \\
\hline 1.50 & 31.6 & 0.032 \\
\hline 1.55 & 35.5 & 0.028 \\
\hline 1.60 & 39.8 & 0.025 \\
\hline 1.65 & 44.7 & 0.022 \\
\hline 1.70 & 50.1 & 0.020 \\
\hline$\cdots:$ & 56.2 & 0.018 \\
\hline 1.80 & 63.1 & 0.016 \\
\hline 1.85 & 70.8 & 0.014 \\
\hline 1.90 & 79.4 & 0.0126 \\
\hline 1.95 & 89.1 & 0.0112 \\
\hline 2.00 & 100 & 0.0100 \\
\hline 2.05 & 112 & 0.0089 \\
\hline 2.10 & 126 & 0.0080 \\
\hline 2.15 & 141 & 0.0071 \\
\hline 2.20 & 159 & 0.0063 \\
\hline 2.25 & 178 & 0.0056 \\
\hline 2.30 & 200 & 0.0050 \\
\hline 2.35 & 224 & 0.0045 \\
\hline 2.40 & 251 & 0.0040 \\
\hline
\end{tabular}




\begin{tabular}{|c|c|c|}
\hline $\begin{array}{c}\text { Density } \\
\text { (Log) }\end{array}$ & $\begin{array}{c}\text { Opacity } \\
\text { (Number) }\end{array}$ & $\begin{array}{c}\text { Transmission or } \\
\text { Reflectance }\end{array}$ \\
\hline 2.45 & 282 & 0.0036 \\
\hline 2.50 & 316 & 0.0032 \\
\hline 2.65 & 447 & 0.0022 \\
\hline 2.75 & 562 & 0.0018 \\
\hline 2.80 & 631 & 0.0016 \\
\hline 2.85 & 708 & 0.0014 \\
\hline 2.90 & 794 & 0.0012 \\
\hline 2.95 & 891 & 0.0011 \\
\hline 3.00 & 1,000 & 0.0010 \\
\hline 4.00 & 10,000 & 0.0001 \\
\hline
\end{tabular}

STEP 5: From Table 2 above, the "black-only" print density readings were more closely aligned with the readings taken from the Kodak gray Card. In fact, gray level values between 125 and 130 were the best match with the average value taken from the gray card. Therefore, the "black-only" printed gray patch images were used for the photographic calibration experiment.

STEP 6: A windowless studio laboratory was used to conduct this experiment. The only permanent lighting in the laboratory was from two rows of fluorescent lights suspended from an off-white textured ceiling. The walls in the laboratory were yellowish cream color.

STEP 7: The gray patch sheets were then mounted on the $18 \%$ gray background wall in three rows of four sheets in each row starting with the lowest reflectivity sheet (100) and

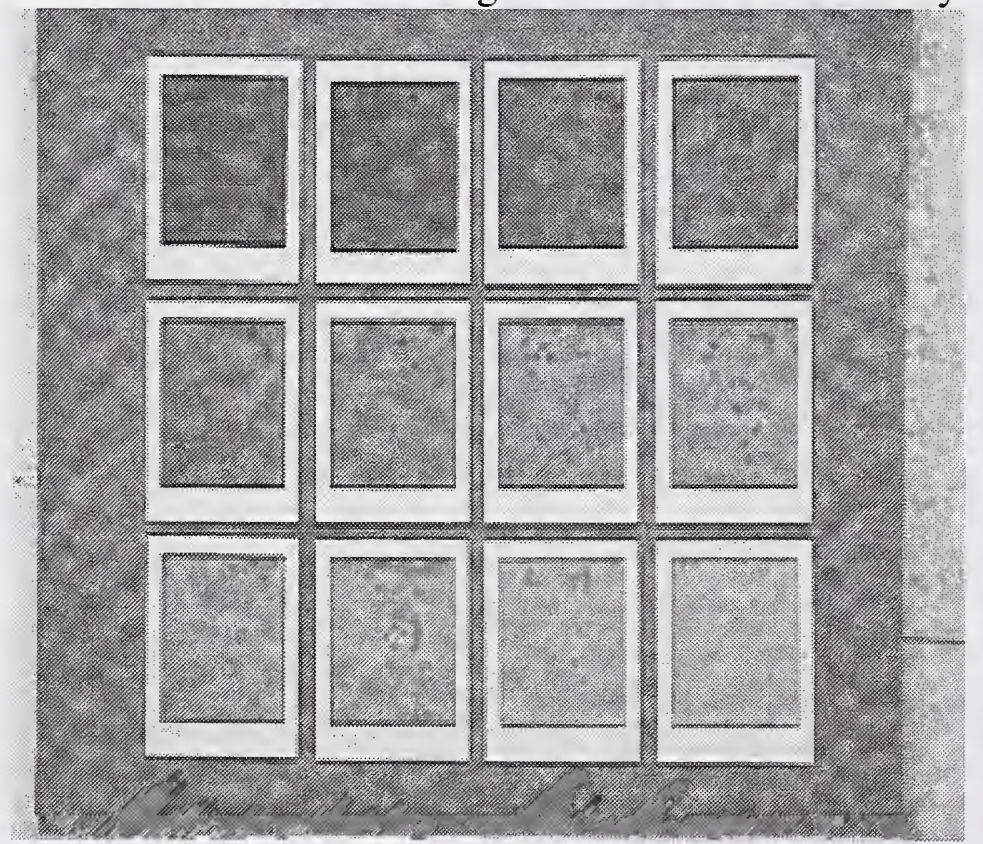

Figure 4- Gray Background Setup 
ending with the highest reflectivity sheet (155) with approximately $2.54 \mathrm{~cm}$ (one inch) of space between each sheet. Figure 4 shows a picture of the backdrop setup with the gray patch

sheets arranged in a three by four array.

STEP 8: Three photoflood lights were used in the experiment. In the initial setup, Sylvania Photo-ECA white bulbs were used with a rating of 250 watts, 120 volts and a luminescence of $3200^{\circ} \mathrm{K}$. The placement of the photoflood lights is shown in Figure 5 below. In the secondary setup, General Electric 115-120 watt blue tinted bulbs were used in place of the white bulbs to reduce the amount of red bias in the digital CCDs within each camera.

STEP 9: The two front floodlights were set six feet off the floor and initially angled inward roughly 15 degrees from the straight to back wall position with no tilt. The center back floodlight was positioned eight feet off the floor and pointed toward the ceiling at an angle of 60 degrees from the horizontal. This was done in an attempt to spread the light evenly over the surface of the backdrop. A light meter was then used to more finely adjust the lights. An attempt was made to get the light meter readings within $\pm 5 \%$ over the backdrop area included in the image frame.

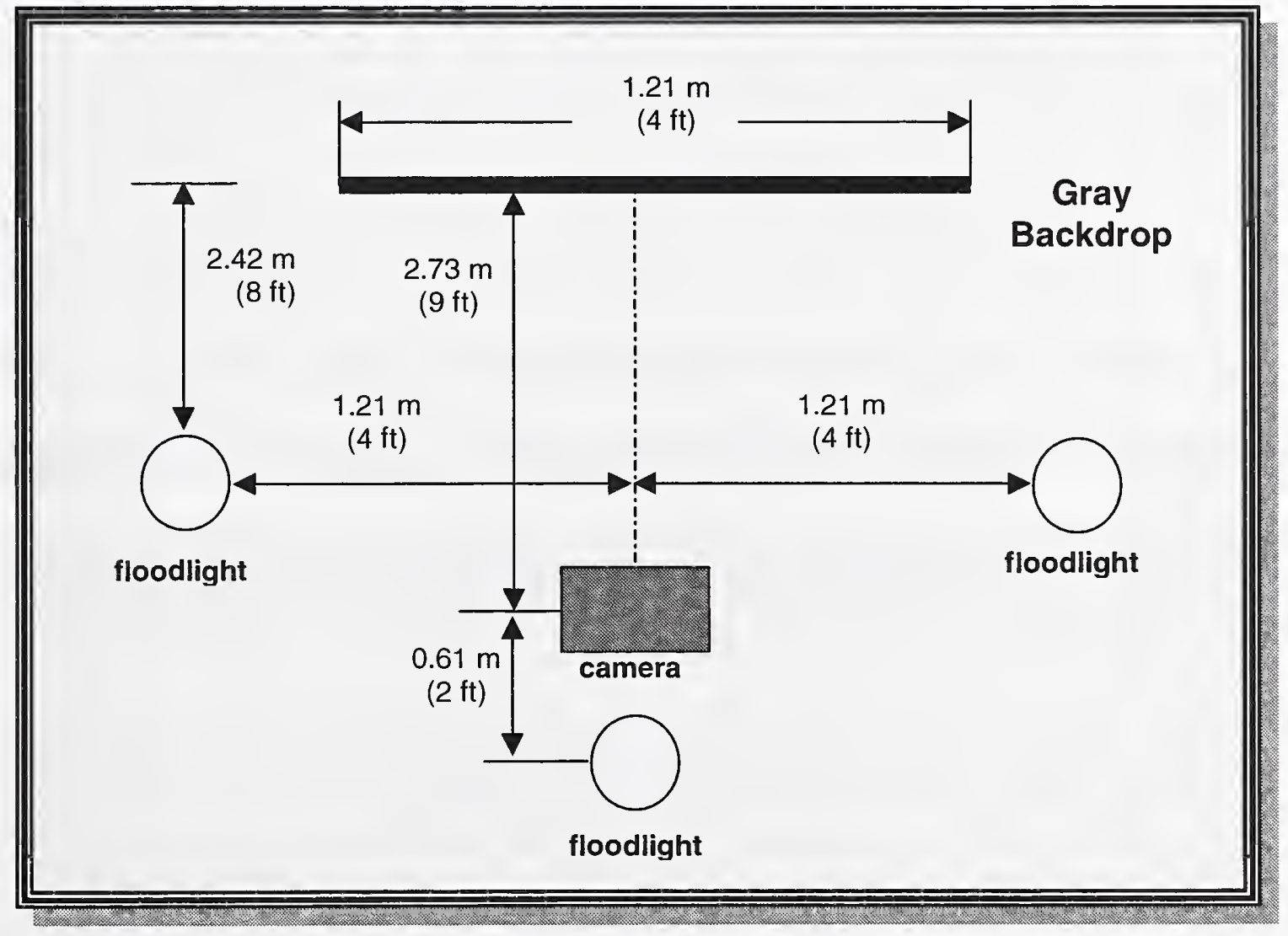


Figure 5 - Mugshot Studio Setup

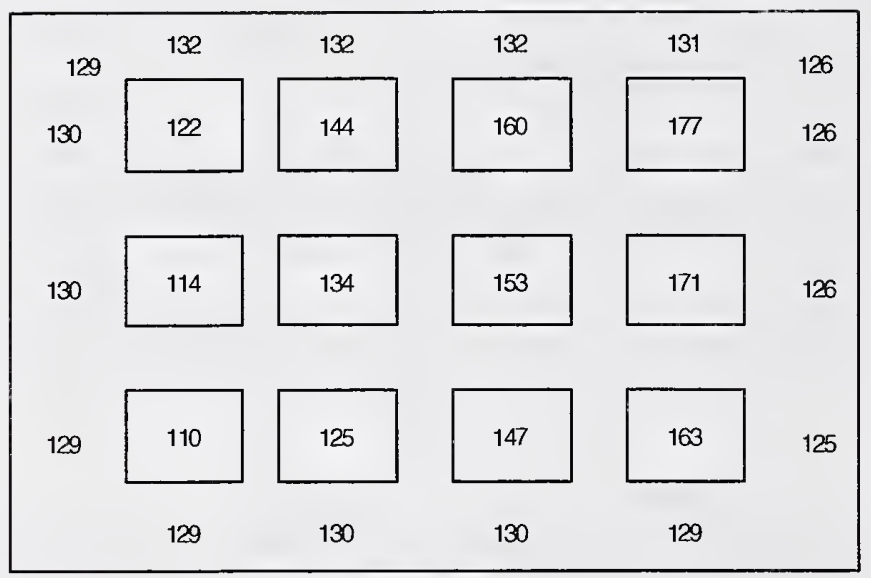

Figure 6 - Gray Level Values with Overhead Lights

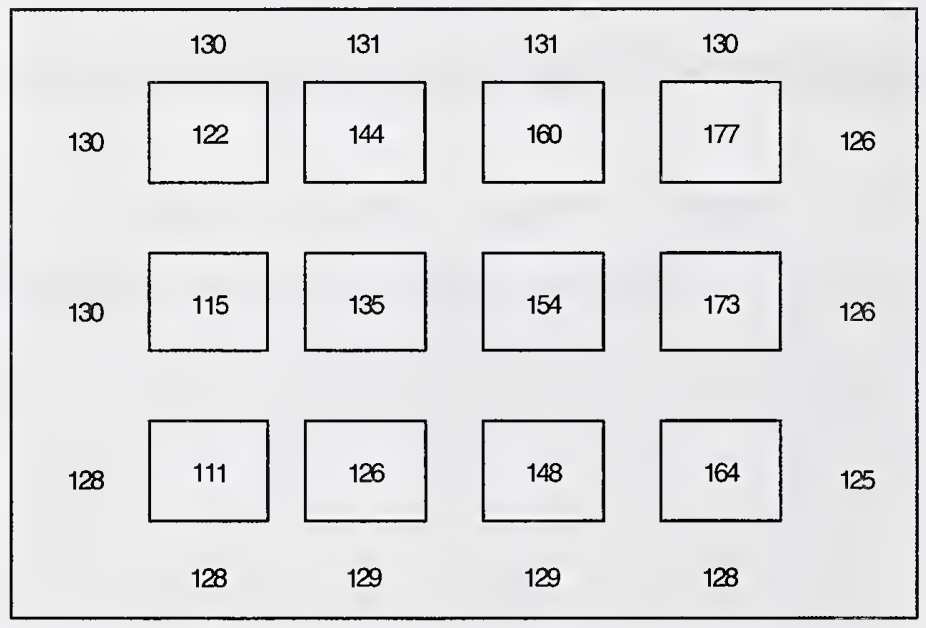

Figure 7 - Gray Level Values without Overhead Lights

STEP 10: Using Camera \#1, set at the exposure value of $1.0 \mathrm{EV}$, Field, the highest quality JPEG compression setting, and zoomed to include the edges of the backdrop, a picture was taken with the floodlights turned on and positioned as described in STEP 9. The resultant picture was then opened in Adobe PhotoShop 4.0 and tested for mean gray level values for each gray patch and various areas of the gray background. Figure 6 illustrates the mean luminosity histogram values taken with the overhead fluorescent lights on. Figure 7 illustrates the mean luminosity histogram values taken with the 
overhead fluorescent lights off.

It is evident from the two figures, shown above, that there was very little difference between the picture without the overhead lights and the picture with the overhead lights. Therefore, one may conclude that overhead lights had no significant effect on the rendered image when the photoflood lights were used. It should also be pointed out that the floodlights had a slight imbalance; i.e., brighter on the left side and darker on the right side. Figures 6 and 7 show a maximum difference of 4 gray level units when comparing the gray level values of the gray background on the left and right sides of the gray patch array. After adjusting the lights by using the light meter, Figures 6 and 7 represent the best settings achieved.

\section{Tests Performed}

As described in the diagram in Figure 5 above, photographs were taken of the twelve gray patch sheets (shown in Figure 4) using the following test scenarios.

\section{Camera \#1, Gray Background Test \#1}

1. Camera \#1 constant camera settings: Frame and Fine (higher resolution than normal)

2. EV (exposure value offset) settings were varied from: -1.5 to +1.5 and varying the steps by 0.5

3. Photoflood lights were positioned as indicated in Step 10 above using the Sylvania Photo-ECA white bulbs with a rating of 250 watts, 120 volts and a luminescence of $3200{ }^{\circ} \mathrm{K}$. All three floodlights were pointed towards the image frame to maximize the light intensity.

Table 3 shows the empirical data results obtained from Adobe's PhotoShop 4.0 for overall gray level intensity values and related values for the red, green and blue channels.

Table 3 - Results for Camera \#1, Test \#1

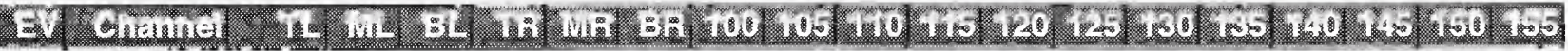

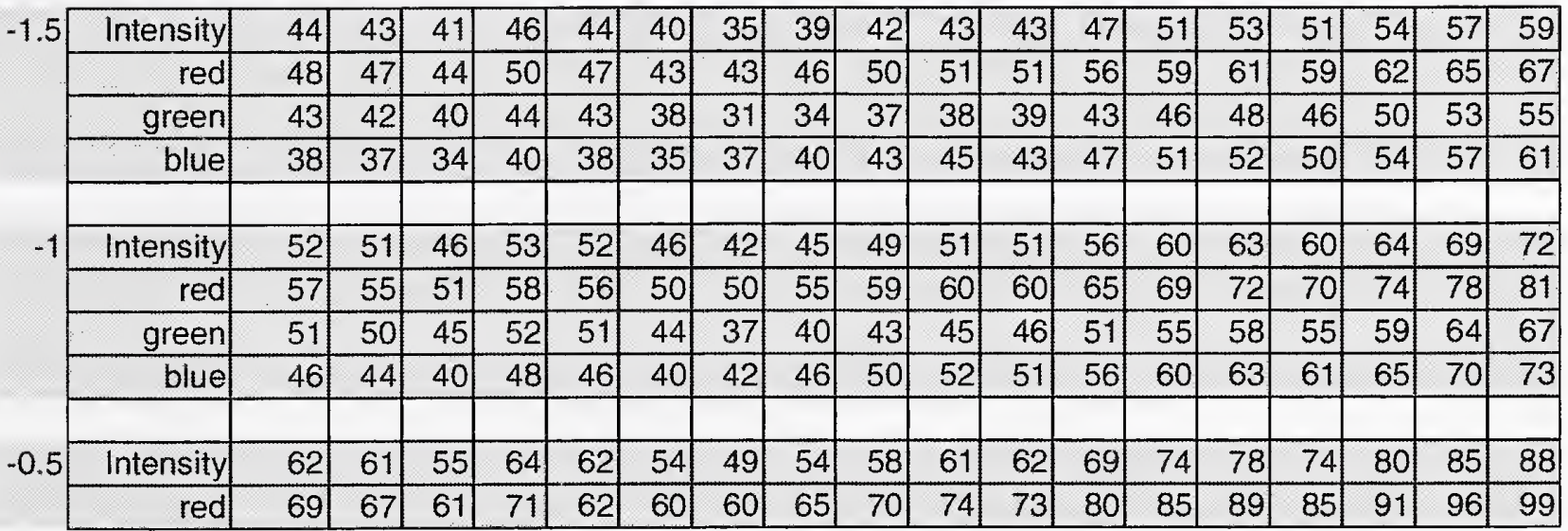


EV Channel TL ML BL TR MR BR 100105110115120125130135140145150155

\begin{tabular}{|r|r|r|r|r|r|r|r|r|r|r|r|r|r|r|r|r|r|r|}
\hline green & 62 & 60 & 54 & 62 & 62 & 53 & 43 & 47 & 52 & 55 & 56 & 63 & 68 & 72 & 68 & 74 & 79 & 83 \\
\hline blue & 56 & 55 & 48 & 58 & 57 & 49 & 43 & 56 & 60 & 64 & 64 & 70 & 74 & 80 & 75 & 82 & 87 & 90 \\
\hline & & & & & & & & & & & & & & & & & & \\
\hline Intensity & 79 & 78 & 69 & 82 & 80 & 70 & 60 & 67 & 74 & 79 & 78 & 86 & 92 & 96 & 92 & 100 & 104 & 109 \\
\hline red & 85 & 83 & 76 & 88 & 86 & 76 & 74 & 82 & 89 & 93 & 92 & 100 & 105 & 110 & 106 & 113 & 118 & 122 \\
\hline green & 77 & 76 & 67 & 80 & 79 & 68 & 53 & 60 & 66 & 71 & 71 & 80 & 85 & 89 & 85 & 93 & 97 & 102 \\
\hline blue & 71 & 71 & 62 & 76 & 74 & 63 & 62 & 68 & 76 & 82 & 80 & 89 & 94 & 100 & 95 & 102 & 108 & 113 \\
\hline
\end{tabular}

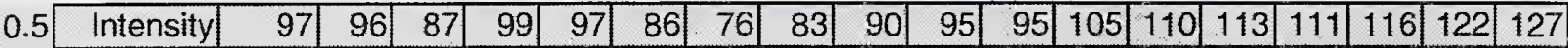

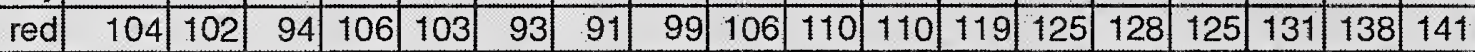

\begin{tabular}{|c|c|c|c|c|c|c|c|c|c|c|c|c|c|c|c|c|c|c|}
\hline green & 95 & 94 & 85 & 97 & 96 & 83 & 68 & 75 & 82 & 87 & 88 & 97 & 102 & 106 & 103 & 109 & 115 & 119 \\
\hline blue & 88 & 86 & 78 & 90 & 89 & 78 & 76 & 85 & 92 & 96 & 95 & 105 & 111 & 116 & 112 & 117 & 123 & 128 \\
\hline & & & & & & & & & & & & & & & & & & \\
\hline Intensity & 115 & 114 & 106 & 117 & 117 & 106 & 94 & 103 & 110 & 113 & 114 & 124 & 132 & 136 & 132 & 141 & 149 & 53 \\
\hline red & 123 & 121 & 114 & 125 & 122 & 113 & 110 & 120 & 127 & 131 & 131 & 140 & 147 & 152 & 148 & 156 & 164 & 169 \\
\hline green & 113 & 112 & 104 & 116 & 115 & 104 & 85 & 94 & 101 & 104 & 105 & 116 & 122 & 128 & 124 & 132 & 140 & 45 \\
\hline blue & 105 & 105 & 96 & 109 & 107 & 96 & 96 & 105 & 112 & 117 & 116 & 125 & 133 & $\overline{139}$ & 133 & $\overline{142}$ & 150 & 156 \\
\hline & & & & & & & & & & & & & & & & & & \\
\hline Intensity & 148 & 148 & 135 & 151 & 151 & 136 & 121 & 132 & 142 & 147 & 148 & 161 & 169 & 181 & 170 & 178 & 183 & \\
\hline red & 157 & 155 & 144 & 161 & 158 & 144 & 141 & 153 & 163 & 168 & 168 & 180 & 189 & 194 & 189 & 197 & 201 & 204 \\
\hline green & 146 & 146 & 133 & 149 & 149 & 134 & 111 & 121 & 130 & 137 & 138 & 151 & 158 & 165 & 160 & 168 & 173 & 176 \\
\hline blue & 135 & 135 & 122 & 139 & 138 & 124 & 123 & 133 & 143 & 150 & 149 & 161 & 169 & 174 & 170 & 176 & 181 & 185 \\
\hline
\end{tabular}

Key:
TL $=$ Top Left Background
$\mathrm{ML}=$ Middle Left Background
$\mathrm{BL}=$ Bottom Left Background
TR = Top Right Background
$\mathrm{MR}=$ Middle Right Background
$\mathrm{BR}=$ Bottom right Background
$\mathrm{EV}=$ Camera $\# 1^{2}$ Exposure Value

\section{Sony Camera \#1, Gray Background Test \#2}

4. Camera \#1 constant camera settings: Frame and Fine (specific settings on camera \#1). The fine setting indicated a JPEC compression setting of approximately 24 to 1 , and the frame setting was an enhanced mode to be used with a tripod.

5. EV settings were varied from: -1.5 to +1.5 and varying the steps by 0.5

6. Photoflood lights were positioned as indicated in Step 10 above using the Sylvania Photo-ECA white bulbs with a rating of 250 watts, 120 volts and a luminescence of $3200^{\circ} \mathrm{K}$.

7. The middle photoflood light was pointed at a 60 -degree angle towards the ceiling to get a more uniform distribution of light across the entire image frame. 
Table 4 shows the empirical data results obtained from Adobe's PhotoShop 4.0 for overall gray level intensity values and intensity values for the red, green and blue channels.

Table 4 - Results for Camera \#1, Test \#2

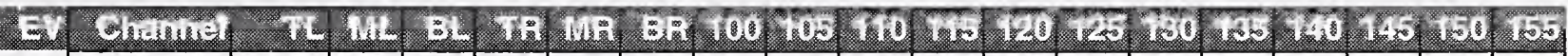

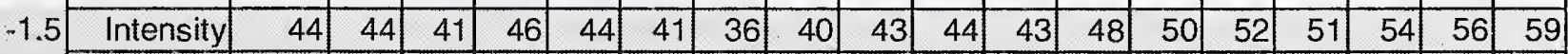

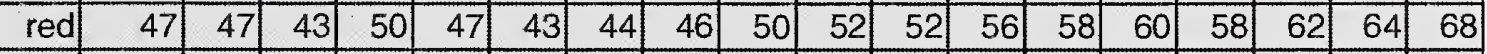

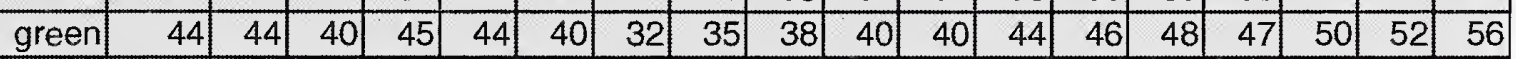

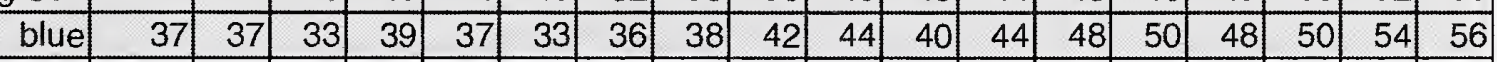

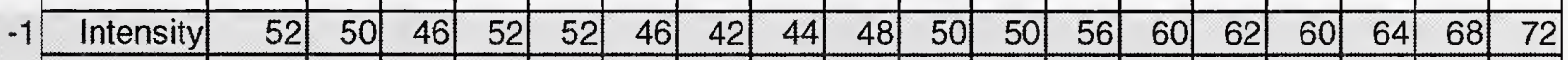

\begin{tabular}{|l|l|l|l|l|l|l|l|l|l|l|l|l|l|l|l|l|l|l|}
\hline red & 58 & 53 & 52 & 58 & 55 & 49 & 50 & 54 & 61 & 63 & 58 & 64 & 71 & 70 & 68 & 72 & 78 & 80 \\
\hline
\end{tabular}

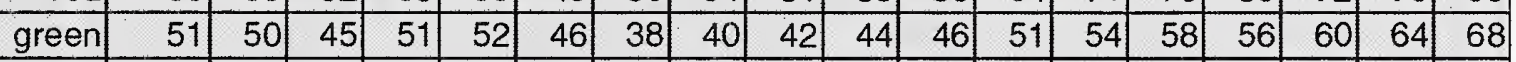
\begin{tabular}{|l|l|l|l|l|l|l|l|l|l|l|l|l|l|l|l|l|l|l|}
\hline blue & 43 & 43 & 39 & 45 & 45 & 39 & 38 & 44 & 48 & 46 & 50 & 52 & 56 & 62 & 56 & 60 & 66 & 68 \\
\hline
\end{tabular}

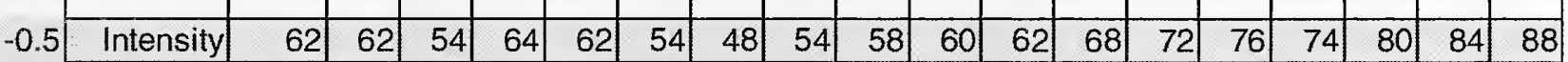
\begin{tabular}{|r|r|r|r|r|r|r|r|r|r|r|r|r|r|r|r|r|r|r|}
\hline red & 68 & 68 & 60 & 70 & 65 & 60 & 61 & 67 & 71 & 73 & 75 & 81 & 85 & 89 & 87 & 93 & 97 & 101 \\
\hline
\end{tabular} \begin{tabular}{|l|l|l|l|l|l|l|l|l|l|l|l|l|l|l|l|l|l|l|}
\hline green & 61 & 61 & 53 & 63 & 62 & 53 & 42 & 48 & 52 & 54 & 56 & 62 & 66 & 70 & 68 & 74 & 78 & 82 \\
\hline
\end{tabular}

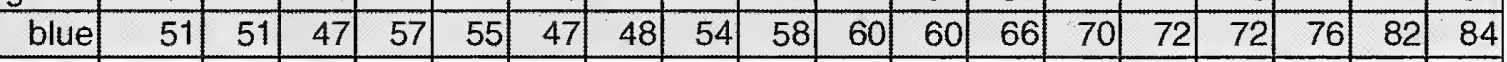

\begin{tabular}{|l|l|l|l|l|l|l|l|l|l|l|l|l|l|l|l|l|l|l|}
\hline Intensity & 80 & 77 & 71 & 82 & 80 & 70 & 61 & 68 & 75 & 78 & 78 & 86 & 92 & 96 & 92 & 99 & 105 & 108 \\
\hline
\end{tabular} \begin{tabular}{|r|r|r|r|r|r|r|r|r|r|r|r|r|r|r|r|r|r|r|}
\hline red & 86 & 84 & 76 & 88 & 86 & 76 & 75 & 81 & 89 & 93 & 93 & 101 & 107 & 109 & 107 & 113 & 119 & 121 \\
\hline
\end{tabular} \begin{tabular}{|l|l|l|l|l|l|l|l|l|l|l|l|l|l|l|l|l|l|l|}
\hline green & 79 & 77 & 69 & 81 & 79 & 69 & 54 & 60 & 67 & 70 & 72 & 79 & 84 & 90 & 86 & 92 & 98 & 101 \\
\hline
\end{tabular} \begin{tabular}{|r|r|r|r|r|r|r|r|r|r|r|r|r|r|r|r|r|r|r|}
\hline blue & 69 & 67 & 61 & 73 & 71 & 61 & 60 & 66 & 70 & 78 & 76 & 84 & 90 & 96 & 90 & 98 & 102 & 106 \\
\hline
\end{tabular}

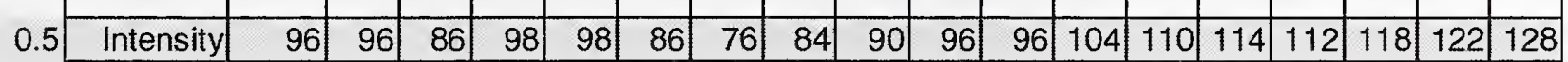
\begin{tabular}{|r|r|r|r|r|r|r|r|r|r|r|r|r|r|r|r|r|r|r|}
\hline red & 103 & 102 & 94 & 106 & 104 & 92 & 91 & 101 & 105 & 111 & 111 & 119 & 125 & 129 & 127 & 133 & 137 & 143 \\
\hline
\end{tabular}

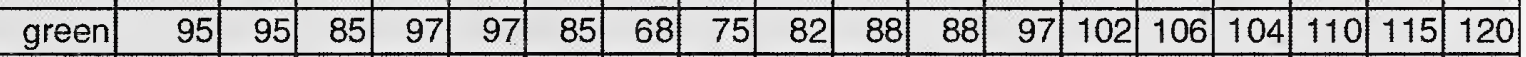

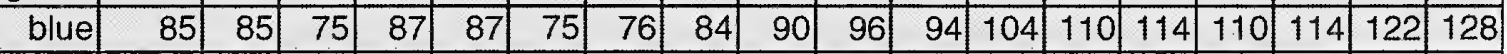

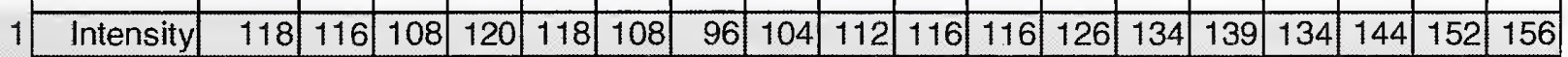

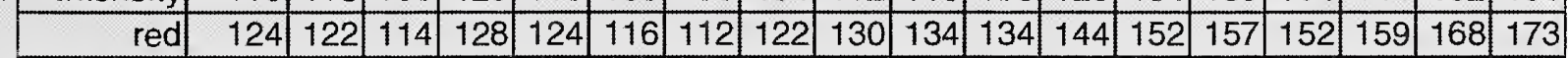

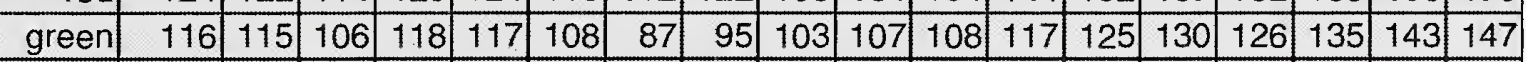
\begin{tabular}{|l|l|l|l|l|l|l|l|l|l|l|l|l|l|l|l|l|l|l|}
\hline blue & 107 & 105 & 97 & 109 & 107 & 97 & 96 & 104 & 112 & 116 & 116 & 126 & 132 & 140 & 134 & 144 & 150 & 156 \\
\hline
\end{tabular}

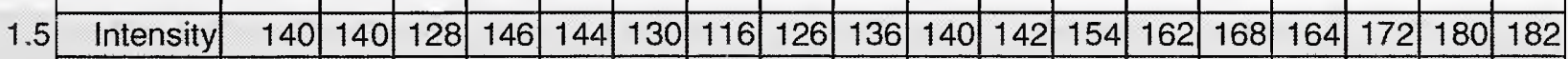
\begin{tabular}{|l|l|l|l|l|l|l|l|l|l|l|l|l|l|l|l|l|l|l|} 
red & 150 & 148 & 138 & 154 & 150 & 138 & 136 & 147 & 157 & 161 & 161 & 174 & 183 & 186 & 182 & 198 & 198 & 200 \\
\hline
\end{tabular} \begin{tabular}{|l|l|l|l|l|l|l|l|l|l|l|l|l|l|l|l|l|l|l|}
\hline green & 138 & 139 & 126 & 143 & 143 & 128 & 105 & 115 & 124 & 129 & 132 & 145 & 151 & 159 & 155 & 163 & 169 & 173 \\
\hline
\end{tabular}

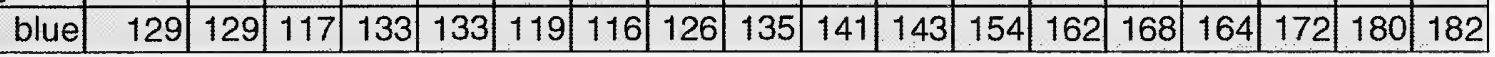




\section{Camera \#1 Gray Background Test \#3}

As a result of the two tests using the twelve separate gray patches, it was found that it was difficult if not impossible to adjust the lighting to be completely uniform over the entire image area. Therefore, a third test was performed using a specially constructed single A4 sized sheet with the twelve gray patches, which was more the size of a human face. By using this methodology, a much more uniform distribution of lighting could be achieved over a much smaller area for the image. As with the twelve separate gray patches, each gray patch on this single sheet varied in gray level intensity values by 5 units ranging from 100 to 155 in gray level intensity. It should also be noted that the red,

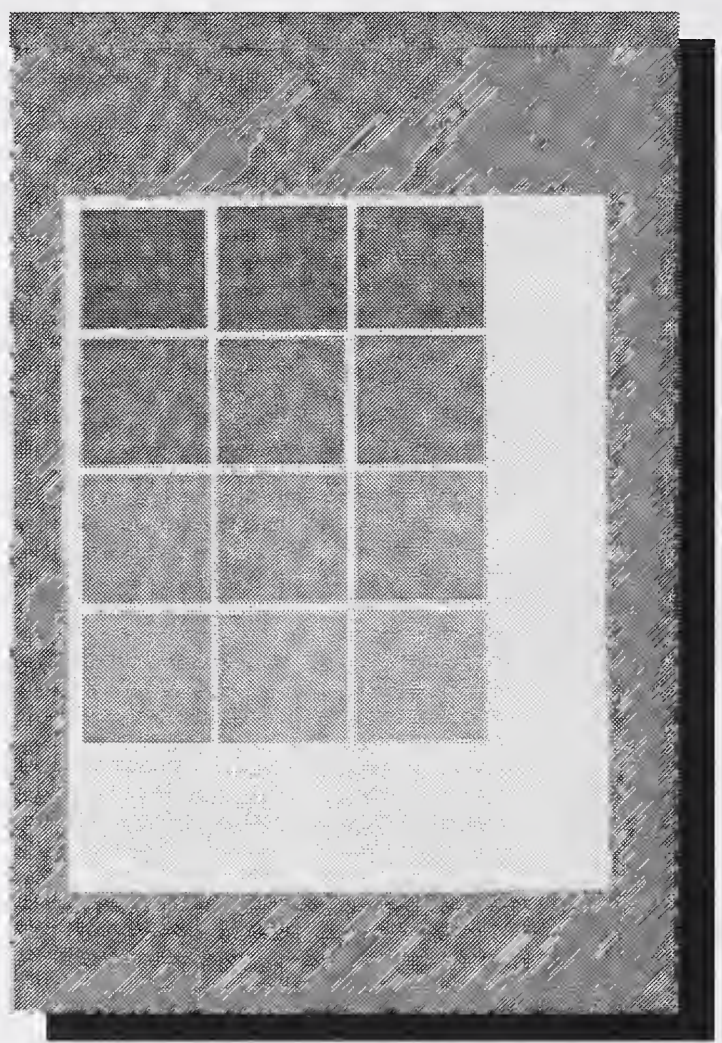

Figure 8 - Single Gray Sheet

green and blue channels were exactly the same as the gray level intensity values for each gray patch. Figure 8 is a picture of the single sheet of gray patches used from this point on in the experiment.

Table 5 gives the results of the Camera \#1 ranging in EV values from 0.0 to 1.5 
Table 5 - Results for Camera \#1, Test \#3

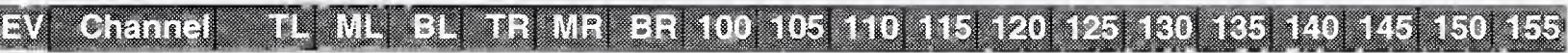

\begin{tabular}{|c|c|c|c|c|c|c|c|c|c|c|c|c|c|c|c|c|c|c|c|}
\hline \multirow[t]{5}{*}{0.0} & Intensity & 83 & 83 & 80 & 79 & 80 & 75 & 65 & 69 & 76 & 78 & 85 & 92 & 93 & 99 & 104 & 105 & 111 & 116 \\
\hline & red & 82 & 82 & 80 & 78 & 79 & 75 & 74 & 77 & 84 & 86 & 92 & 99 & 100 & 105 & 110 & 111 & 116 & 121 \\
\hline & green & 84 & 85 & 81 & 81 & 82 & 77 & 61 & 66 & 72 & 75 & 82 & 89 & 91 & 97 & 102 & 104 & 110 & 115 \\
\hline & blue & 72 & 73 & 70 & 71 & 71 & 66 & 60 & 65 & 71 & 74 & 80 & 87 & 88 & 94 & 99 & 100 & 106 & 111 \\
\hline & & & & & & & & & & & & & & & & & & & \\
\hline \multirow[t]{5}{*}{0.5} & Intensity & 101 & 102 & 98 & 95 & 98 & 93 & 82 & 87 & 94 & 97 & 105 & 111 & 113 & 118 & 124 & 126 & 132 & 139 \\
\hline & red & 101 & 101 & 98 & 95 & 95 & 92 & 92 & 98 & 104 & 105 & 113 & 119 & 120 & 125 & 131 & 131 & 138 & 145 \\
\hline & green & 103 & 104 & 100 & 98 & 100 & 94 & 79 & 83 & 90 & 94 & \begin{tabular}{|l|}
101 \\
\end{tabular} & 109 & 110 & 116 & 122 & 124 & 131 & 138 \\
\hline & blue & 90 & 91 & 87 & 86 & 87 & 82 & 77 & 82 & 89 & 91 & 99 & 106 & 107 & 113 & 119 & 120 & 127 & 133 \\
\hline & & & & & & & & & & & & & & & & & & & \\
\hline \multirow[t]{5}{*}{1.0} & Intensity & 122 & 123 & 120 & 114 & 118 & 113 & 103 & 108 & 114 & 118 & 126 & 135 & 137 & 145 & 152 & 154 & 160 & 168 \\
\hline & red & 123 & 123 & 121 & 115 & 118 & 113 & 117 & 122 & 127 & 130 & 138 & 147 & 148 & 156 & 163 & 163 & 169 & 177 \\
\hline & green & 123 & 125 & 121 & 116 & 120 & 114 & 98 & 103 & 109 & 113 & 121 & 130 & 133 & 140 & 148 & 150 & 157 & 165 \\
\hline & blue & 108 & 110 & 107 & 103 & 106 & 101 & 97 & 102 & 108 & 111 & 119 & 129 & 130 & 137 & \begin{tabular}{|l|}
145 \\
\end{tabular} & 147 & 153 & 161 \\
\hline & & & & & & & & & & & & & & & & & & & \\
\hline \multirow[t]{4}{*}{1.5} & Intensity & 149 & 153 & 146 & 139 & 146 & 138 & 128 & 135 & 144 & 148 & 158 & 167 & 169 & 177 & 182 & 182 & 187 & 192 \\
\hline & red & 154 & 159 & 152 & 144 & 150 & 142 & 148 & 154 & 163 & 166 & 175 & 185 & 186 & 193 & 198 & 198 & 202 & 206 \\
\hline & green & 148 & 154 & 146 & 139 & 147 & 138 & 120 & 126 & 135 & 141 & 151 & 160 & 162 & 170 & 175 & 176 & 181 & 186 \\
\hline & blue & 134 & 138 & 130 & 126 & 132 & 120 & 120 & 127 & 136 & 140 & 149 & 159 & 161 & 168 & 174 & 175 & 178 & 183 \\
\hline
\end{tabular}

\section{Camera \#2 Gray Background Tests}

Using the same lighting configuration as described above for Camera \#1 tests, Camera \#2 with a pixel resolution setting of the maximum 1024 by 950 pixel resolution was used. Pictures were taken of the gray patch backdrop while varying the exposure setting from a minimum of -2 to a maximum of +2 in increments of 0.5 exposure steps. Table 6 below shows the values obtained when the resultant images were analyzed using the PhotoShop 4 software, and utilizing the histogram function for determining the overall gray level as well as the values for each of the RGB channels.

Table 6 - Results for Camera \#2 Test

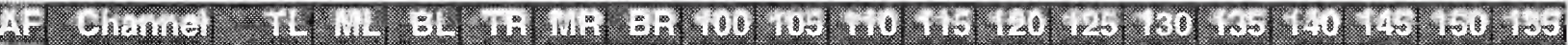

\begin{tabular}{|c|c|c|c|c|c|c|c|c|c|c|c|c|c|c|c|c|c|c|c|}
\hline & Intensity & 52 & 50 & 46 & 52 & 52 & 46 & 42 & 44 & 48 & 50 & 50 & 56 & 60 & 62 & 60 & 64 & 68 & 72 \\
\hline & red & 58 & 53 & 52 & 58 & 55 & 49 & 50 & 54 & 61 & 63 & 58 & 64 & 71 & 70 & 68 & 72 & 78 & 80 \\
\hline & green & 51 & 50 & 45 & 51 & 52 & 46 & 38 & 40 & 42 & 44 & 46 & 51 & 54 & 58 & 56 & 60 & 64 & 68 \\
\hline & blue & 43 & 43 & 39 & 45 & 45 & 39 & 38 & 44 & 48 & 46 & 50 & 52 & 56 & 62 & 56 & 60 & 66 & 68 \\
\hline & Intensity & 62 & 62 & 54 & 64 & 62 & 54 & 48 & 54 & 58 & 60 & 62 & 68 & 72 & 76 & 74 & 80 & 84 & 88 \\
\hline
\end{tabular}


AF Channel TL ML BL TR MR BR 100105110115120125130135140145150155

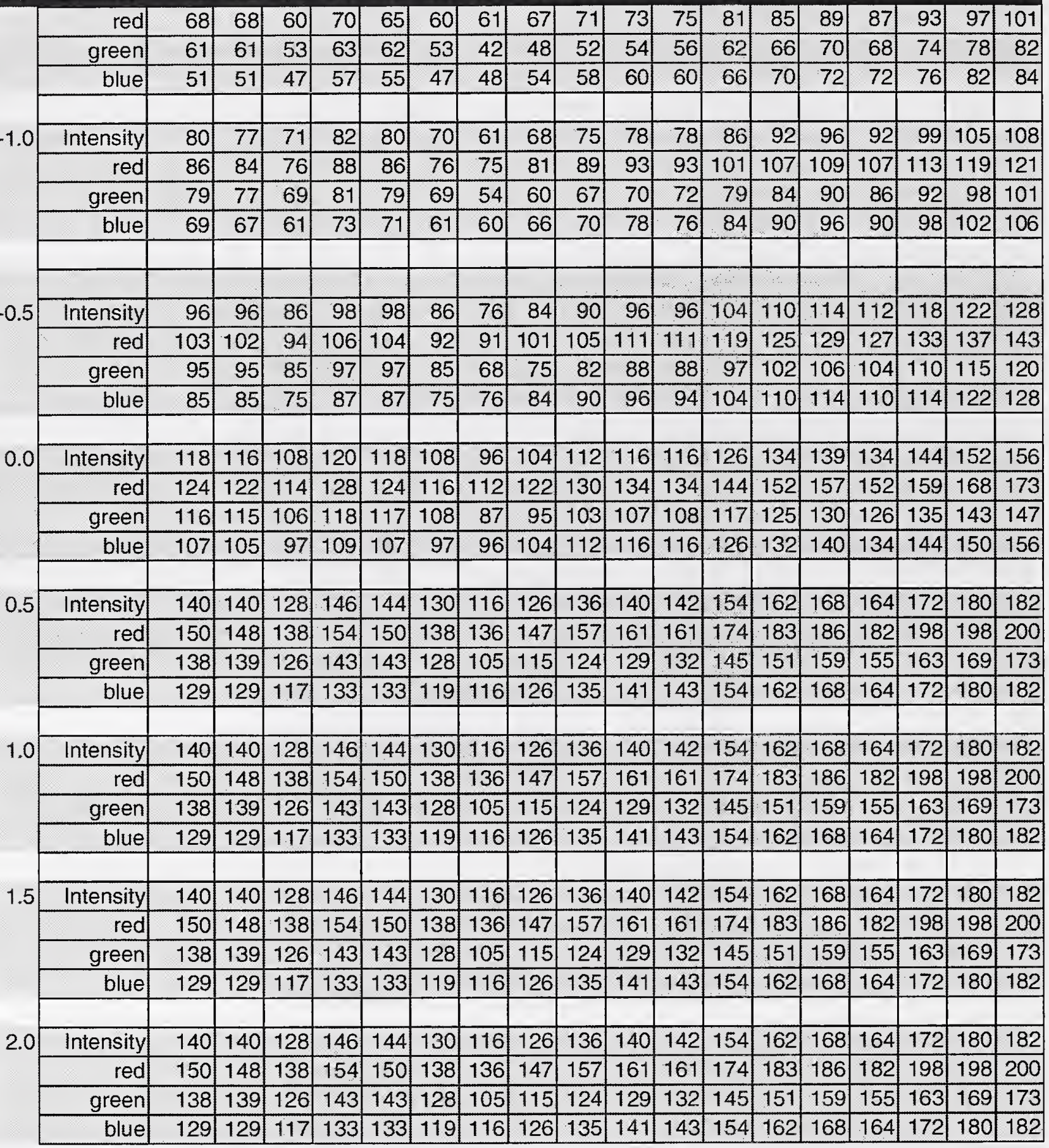

\section{Camera \#3 Gray Background Test with Flood Lamps}

Again, using the same lighting configuration as described for both Camera \#1 and Camera \#2 tests, Camera \#3 with a pixel resolution of 1500 by 1200 was used. Pictures were taken of the gray patch backdrop while varying the exposure setting manually by using a combination of shutter speed and f-stop until the 125-gray patch had a value close 
to 125 as possible without changing the lighting configuration. The table below shows the values obtained when the resultant images were analyzed using the PhotoShop 4 software, and utilizing the luminosity histogram function for determining the overall gray level as well as the values for each of the RGB channels.

Table 7 - Results for Camera \#3 Test

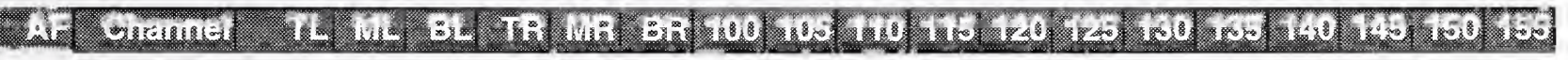

\begin{tabular}{|c|c|c|c|c|c|c|c|c|c|c|c|c|c|c|c|c|c|c|c|}
\hline \multirow[t]{5}{*}{$1 / 125$} & Intensity & 65 & 66 & 60 & 55 & 55 & 52 & 50 & 54 & 61 & 63 & 72 & 78 & 78 & 87 & 91 & 90 & 97 & 103 \\
\hline & red & 69 & 71 & 65 & 60 & 62 & 57 & 63 & 68 & 74 & 74 & 85 & 93 & 91 & 101 & 105 & 102 & 110 & 115 \\
\hline & green & 65 & 65 & 60 & 54 & 54 & 50 & 45 & 49 & 55 & 58 & 66 & 72 & 72 & 81 & 85 & 84 & 92 & 98 \\
\hline & blue & 58 & 56 & 51 & 47 & 47 & 44 & 48 & 52 & 60 & 61 & 67 & 74 & 75 & 82 & 86 & 85 & 92 & 97 \\
\hline & & & & & & & & & & & & & & & & & & & \\
\hline \multirow[t]{4}{*}{$1 / 30$} & Intensity & 125 & 120 & 122 & 122 & 119 & 121 & 99 & 104 & 109 & 115 & 120 & 128 & 132 & 138 & 144 & 148 & 154 & 161 \\
\hline & red & 158 & 147 & 151 & 156 & 144 & 149 & 131 & 135 & 142 & 149 & 155 & 162 & 168 & 173 & 180 & 184 & 191 & 198 \\
\hline & green & 108 & 106 & 107 & 104 & 105 & 106 & 82 & 86 & 91 & 96 & 100 & 109 & 113 & 118 & 123 & 128 & 134 & 140 \\
\hline & blue & 127 & 125 & 123 & 126 & 126 & 124 & 109 & 115 & 119 & 123 & 129 & 136 & 140 & 146 & 152 & 157 & 163 & $\overline{171}$ \\
\hline
\end{tabular}

We observed from Table 7 above that the exposure setting of 1/125 sec at F4 with the photo lamps in the same position as in the other camera tests was not sufficient for obtaining the correct gray readings. However, the shutter setting of $1 / 30 \mathrm{sec}$ most closely matched the gray calibration sheet. Notice that the overall illumination measurements came very close to the actual numbers. It was further observed that the red channel gave much higher readings than either the green or the blue channels. We also observed that the blue channel came closest to the overall illumination readings. The net result was that the image appeared to have a red bias, which to the eye appeared to be tinted pink.

When comparing camera \#3 with both camera \#2 and camera \#1, it was evident that the exposure could be more tightly controlled through the use of both aperture settings and shutter speed settings. However, the other two cameras could only be controlled through the one overall EV setting. When using Camera \#3, the only way to achieve close approximations to the gray calibration sheet would be to use a filter that would align the red channel to the other two color channels, thus eliminating the red bias.

\section{Subject Exposure Tests}

The objective of the "variation in clothing" tests were to show how the individual cameras reacted in exposure compensation to subjects wearing black clothing compared to subjects wearing lighter clothing. Even though each camera was first calibrated to the $18 \%$ gray background using a given photo flood lighting situation, the subject's clothing 
made a difference in exposure for both the camera \#1, camera \#2, and the automatic setting on camera \#3. The electronic circuitry within each of those cameras compensated for the variance in clothing thus producing the effect of changing the gray level values in the gray background. Unfortunately, neither camera \#1 or camera \#2 had any manual override settings that would keep the background exposure constant. Only camera \#3 had the capability to override the electronic compensating factors due to a variance in clothing.

There were three series of tests performed; i.e., one test for each of the three cameras. The gray level values for each of these tests are shown in Tables 9, 10, and 11.

\section{Camera \#1 Clothing Test}

The gray background was first shot at an EV 0.0 setting without a subject in the frame. The gray level value for the gray background image yielded a measurement of 118 with the PhotoShop luminosity histogram function. Then the subject was introduced to the frame wearing samples of light and black clothing. The results are shown in Table 8 for camera \#1. In all instances with the subject in the frame, the measured luminescence of the background was lighter than when there was no subject in the frame. Furthermore, the black clothing produced still lighter measurements than the lighter clothing.

\section{Table 8 - Camera \#1 Clothing Test Results with EV =0.0}

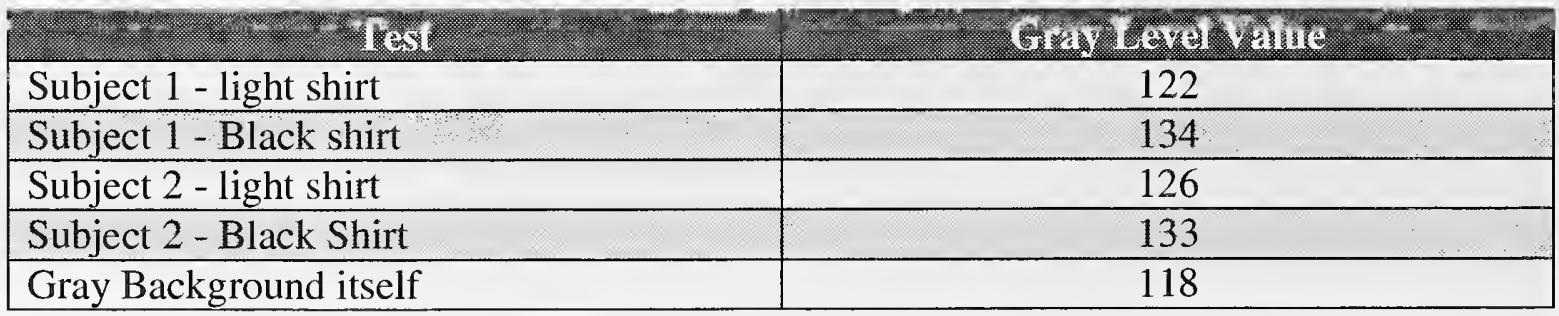

\section{Camera \#2 Clothing Test}

The above test was repeated using camera \#2. Camera \#2 was set at an EV value of 1.0 to obtain measurements in the range of 110 to 130 for the gray background. The results as shown in Table 9 were very similar to the camera \#1 clothing test, i.e., the subjects wearing black measured the highest values for the gray background. 
Table 9 - Camera \#2 Clothing Test Results

\begin{tabular}{|l|c|}
\hline \multicolumn{1}{|c|}{ Test } & 119 \\
\hline Subject 1 - light shirt & 136 \\
\hline Subject 1 - Black shirt & 115 \\
\hline Subject 2 - light shirt & 129 \\
\hline Subject 2 - Black Shirt & 113 \\
\hline Gray Background itself & \\
\hline
\end{tabular}

\section{Camera \#3 with Manual Settings}

The same test was again repeated for camera \#3. The camera was set at $1 / 30 \mathrm{sec}$ with an aperture setting of f4.5. Also, the ASA setting was at 400. The gray background image was measured at 124, which just about matched the value of the Kodak gray card. The results (Table 10) of this test were different from the tests with the other two cameras. The subjects with the light colored shirts yielded a measured background value below the background image value, but the black shirt value was in concordance with the other two cameras. The variance only indicates a difference in sensitivities of the light metering system of camera \#3 when compared with both camera \#1 and camera \#2.

Table 10 - Camera \#3 Clothing Test Results

\begin{tabular}{|l|c|}
\hline \multicolumn{1}{|c|}{ Trest } & - \\
\hline Subject 1 - light shirt & 104 \\
\hline Subject 1 - Black shirt & 135 \\
\hline Subject 2 - light shirt & 116 \\
\hline Subject 2 - Black Shirt & 130 \\
\hline Gray Background itself & 124 \\
\hline
\end{tabular}

\section{Obtained Results}

\section{Camera \#1, Test 1 Results:}

The twelve separate gray patch photoflood test showed the following results:

1. The illumination of the background was uneven, especially with the gray level intensity values taken at the top of the frame when compared with values taken at the bottom of the frame. Also, the values on the right side were higher than the values on the left side. Detailed data is available upon request.

2. Values were then plotted for the gray level values taken inside of each gray patch. The significant points to be made from these plots are as follows: 
- Camera \#1 showed a definite red bias as indicated by the constantly higher values obtained for the red channel. This indicates that the recorded gray surfaces had a slightly reddish tint.

- The blue channel was measured to be the closest to the values obtained for the overall gray level intensity values.

- The green channel was measured to be slightly lower in values than both the red and blue channels.

- From the tests taken with the densitometer, it was shown that the gray patch with the intensity value of 125 most closely matched the Kodak Gray Card by having a density value of 0.76 (the Kodak card had a density value of 0.75 ). Therefore, the camera \#1, Test \#1 results showed that the set of values for EV setting of 1.0 were the closest to the $18 \%$ gray requirement; i.e., the values obtained for the "125 intensity gray patch" were 124 for overall gray level intensity and 140,116, and 125 for RGB respectively.

- As the EV setting was increased to the maximum exposure of 1.5 the red channel was very exaggerated when compared to the other EV settings. The blue channel was still the best in following the overall gray level values.

\section{Camera \#1, Test 2 Results:}

These results were not significantly different from the results obtained for camera \#1, test \#1. When comparing the results between camera \#1, test \#1 and camera \#1, test \#2, one can deduce that the lighting was much more uniform in the camera \#1, test 2 but still uneven from top to bottom.

\section{Camera \#2 Test Results}

Camera \#2 test used the same lighting setup as in the camera \#1 tests. The single sheet of twelve gray patches was used, since it rendered a much more uniform lighting situation. Images were produced varying the camera EV settings from -2 to +2 in increments of 0.5. The best results were achieved at EV 0 setting as shown in Table 6 above. The overall intensity for the "125" gray patch was measured to be 126 . The blue channel was also measured to be 126 . The red channel was much higher and the green channel was a little lower. It was clearly evident that the camera had a red bias as seen from the obtained measurements.

\section{Conclusions and Inferences}

The following points are made from the review of tests and associated cameras used in the experiments:

1. When calibrating a camera to match the $18 \%$ gray backdrop, the use of a single sheet with gray patches should be employed so that the lighting will be uniformly distributed over the entire surface.

2. Exposure compensations should be made for individual subject conditions of clothing, skin texture hair coloring, etc. 
3. Digital camera models that do not have manual exposure override features are less adaptable for the mug shot process.

4. Cameras having manual controls should be used in order to get a uniform exposure values for the gray background.

5. Subjects wearing different colored clothing will affect the outcome of the exposure on automatically controlled digital cameras.

6. As the exposure setting is increased to the maximum, the variance between the red, blue and green channels is exaggerated, thus making the gray background look tinted in nature. Use enough lighting to be able to set the camera at the 0.0 exposure setting and still obtain an $18 \%$ gray rendering of the background. 


